\title{
Activités
}

1-1 | avril 2004

Varia

\section{Formes et enjeux de l'analyse de l'activité pour la conception des systèmes de travail}

\section{Pascal Béguin et Marianne Cerf}

\section{(2) OpenEdition}

\section{Journals}

Édition électronique

URL : http://journals.openedition.org/activites/1156

DOI : 10.4000/activites. 1156

ISSN : 1765-2723

\section{Éditeur}

ARPACT - Association Recherches et Pratiques sur les ACTivités

\section{Référence électronique}

Pascal Béguin et Marianne Cerf, «Formes et enjeux de l'analyse de l'activité pour la conception des systèmes de travail », Activités [En ligne], 1-1 | avril 2004, mis en ligne le 02 avril 2004, consulté le 10 décembre 2020. URL : http://journals.openedition.org/activites/1156; DOI : https://doi.org/10.4000/ activites. 1156

\section{(c) (i) (9)}

Activités est mis à disposition selon les termes de la licence Creative Commons Attribution - Pas d'Utilisation Commerciale - Pas de Modification 4.0 International. 


\title{
Formes et enjeux de l'analyse de l'activité pour la conception des systèmes de travail
}

\author{
Pascal Béguin \\ CNAM, Laboratoire d'Ergonomie, \\ 41 rue Gay Lussac, 75005 Paris \\ beguin@cnam.fr \\ Marianne Cerf \\ INRA, UMR SAD-APT, \\ Bâtiment EGER, BP1, 78850 Thiverval-Grignon \\ cerf@inapg.inra.fr
}

\begin{abstract}
RÉSUMÉ
Dans la perspective d'une ergonomie pour la conception, nous considérons qu'être acteur de la conception, c'est bien sûr analyser l'activité, mais également faciliter sa prise en compte en faisant des propositions qui peu ou prou modifient la conduite du projet, et les activités de conception. Nous défendons ici la thèse qu'il existe différentes manières de penser et de définir le statut et les enjeux de l'analyse de l'activité de travail des opérateurs pour la conception. Cet article est ainsi une tentative, sans doute partiale et partielle, de clarification, d'analyse et de classification de ces manières de référer à l'activité pour la conception. Nous identifions trois postures que l'ergonome peut adopter pour analyser l'activité pour la conception. Il peut chercher à anticiper l'action en situation. Il peut favoriser la conception de dispositifs plastiques, qui orientent (favorisent mais contrôlent) l'action in situ. Il peut encore chercher à saisir et articuler dans un même mouvement le développement des situations de travail et le développement de l'activité des opérateurs. Ces trois postures conduisent à des compréhensions différentes de l'acte de conception en particulier de sa saisie temporelle et dans la construction sociale nécessaire à l'inscription du travail de l'ergonome dans le processus de conception.
\end{abstract}

MOTS CLÉ

outils et techniques pour la conception, analyse de l'activité, méthodes ergonomiques, conception participative.

\begin{abstract}
From our perspective, to work as an ergonomist for the design of working systems, not only means to analyse the activity but also means to facilitate its inclusion in the design process through proposals which might result in changes in the monitoring of the project and in the design activities. In this article, we will argue that different ways can be chosen to think and define the status and the stakes of the analysis of workers' activity for the design process. Our purpose is to start to clarify and classify these different ways from our own and probably limited perspective. Through literature review and our own experience, we identify three main positions: the ergonomist
\end{abstract}


can try to anticipate the workers' activity in their working situations. He (she) can support the design of flexible devices which direct (support and control) the action in situ. He (she) can try to catch and articulate in a same process the development of both the situation and the activity. These different positions result in different understanding of the design process mainly in its temporal dimension. They also result in different ways of building the social relations and the place of the ergonomist within the design project.

\section{KEYWORDS}

tools and techniques for system development, activity analysis, ergonomic methods, cooperative design.

\section{1.- Introduction}

L'ergonomie de conception se développe en explorant deux thématiques. La première, l'analyse de l'activité dans la conception, vise à comprendre le travail des acteurs de la conception ${ }^{1}$. Tout un ensemble de domaines (gestion, psychologie, sociologie, ...) considère qu'une meilleure connaissance des activités et des processus de conception est une nécessité, étant donné les enjeux qu'ils véhiculent pour l'efficacité des industries et pour leur compétitivité (Terssac, de \& Frieberg, 1996). L'analyse du travail des concepteurs contribue au développement de cet axe de recherche interdisciplinaire. La seconde thématique, qu'on qualifiera d'analyse de l'activité pour la conception, vise la conception des systèmes techniques ou des organisations futures à partir de l'analyse de l'activité des «opérateurs finaux », c'est-à-dire de ceux qui mettront en œuvre in fine le dispositif pour atteindre les performances requises ${ }^{2}$.

Bien que distincts, ces deux axes de recherche et d'intervention se fécondent mutuellement. Ainsi, l'analyse de l'activité dans la conception contribue à fournir des repères dont l'ergonome doit tenir compte pour la conception des situations de travail (Falzon, 1995 ; Garrigou, 1995 ; Béguin \& Darses, 1998). Dans cet article, nous nous intéressons à la relation inverse. Les processus de conception ne sont que rarement pensés de façon à prendre en compte le travail et l'activité de travail. Dans ce contexte, être acteur de la conception, c'est bien sûr analyser l'activité, mais également faciliter sa prise en compte en faisant des propositions qui peu ou prou modifient la conduite du projet, et les activités de conception. C'est même ce mouvement qui fonde, de notre point de vue, une ergonomie pour la conception : la capacité à faire des propositions opératoires de conception alternatives, plus favorables à la prise en compte de l'activité des femmes et des hommes.

Or, il existe différentes manières de penser et de définir le statut et les enjeux de l'activité de travail des opérateurs pour la conception. C'est en tout cas la thèse que nous défendrons dans cet article. Nous pensons qu'un travail de clarification, d'analyse et de classification de ces manières de référer à l'activité pour la conception est souhaitable. En effet, ces différences constituent autant d'arrièreplans, à partir desquels sont construits des manières d'agir, des théories, des méthodes, voire des programmes de recherche.

C'est donc à cet exercice (risqué !) que nous nous essayons dans cet article. À partir de notre propre compréhension, et au risque de forcer le trait, nous avons choisi de présenter ces arrière-plans sous la forme de principes. Nous en distinguerons trois. Le premier principe pose la nécessité d'une anti-

1. Concepteurs professionnels, mais aussi d'autres catégories professionnelles impliquées dans la conduite d'un projet (financement, arbitrages multiples, chantier, etc). Dans la suite de ce texte, nous utiliserons le terme générique de « concepteur » pour parler des acteurs de la conception.

2. Dans la suite de ce texte, on remplacera le terme « d'opérateurs finaux » par « opérateurs ». 
cipation de l'activité, et affirme que cette anticipation devrait être partie intégrante des stratégies de conception. Le second postule que l'activité en situation permet de rendre les situations conçues plus efficaces, et préconise une plasticité des systèmes techniques ou des organisations. Le troisième principe appréhende la conception comme un processus développemental, où caractéristiques des situations et activités de travail évoluent dialectiquement durant la conduite du projet. Chaque principe donne éventuellement lieu à une diversité de démarches et d'axes de recherche. Sans prétendre être exhaustif, nous en présenterons quelques-uns. Nous nous attarderons un peu plus sur le troisième, dans lequel se situe notre propre activité de recherche.

\section{2.- Anticiper l'activité future}

Le premier principe peut être formulé de la manière suivante : une anticipation du fonctionnement de l'homme et de son activité est partie intégrante du processus de conception. En effet tout dispositif technique, tout artefact, mobilise durant sa conception une connaissance, une représentation, et au sens le plus large un modèle de l'utilisateur et de son activité.

Ceci est vrai même lorsque l'ergonome est absent du processus de conception. Dans un travail récent, Nicolas (2000) montre ainsi que les concepteurs de systèmes d'aide à la conduite automobile réalisent des simulations langagières ${ }^{1}$ de l'activité de conduite. Celles-ci visent à anticiper les usages des dispositifs qu'ils conçoivent afin d'en préciser les caractéristiques. Deux stratégies complémentaires sont utilisées durant ces simulations. La première consiste à prêter aux utilisateurs des caractéristiques à partir d'une réflexion basée sur le «bon sens»; dans la seconde, les concepteurs s'identifient aux usagers. Ces deux stratégies sont évidemment limitées. La première parce qu'elle prête a priori certaines caractéristiques aux conducteurs. La seconde parce que les concepteurs sont des experts dans le domaine des systèmes embarqués, ce qui n'est pas le cas des futurs usagers. Or de telles anticipations, qui seront matérialisées, puis véhiculées dans les situations de travail par l'artefact ou le système, sont sources de difficultés, voire d'exclusion pour les personnes, lorsqu'elles reposent sur des modèles faux ou insuffisants. Prévoir des escaliers pour accéder aux locaux repose sur la représentation de sujets valides, qui exclut des personnes en fauteuil roulant : ces derniers ne pourront pas accéder à l'étage.

Quelles sont les pratiques ergonomiques auxquelles correspond ce premier principe ? Globalement, il conduit à l'idée, bien résumée par Thomas et Kellogs (1989) qu'à défaut d'une anticipation suffisante de l'Homme et de son activité, le processus de conception est « lacunaire ». Pour combler cette lacune, l'activité de l'intervenant doit se développer au moins sur deux plans : se situer au sein d'un collectif d'acteurs d'une part, et mobiliser des méthodes aptes à y faire des propositions opératoires d'autre part.

Etre acteur du processus de conception implique d'accepter de se confronter à des points de vue alternatifs car le processus de conception met aux prises un ensemble hétérogène d'acteurs : quel que soit l'objet à concevoir (situation de travail, ouvrage d'art, objet divers, etc...) celui-ci est trop complexe pour qu'une seule personne dispose de toutes les compétences nécessaires à la résolution de tous les problèmes. Les acteurs doivent donc coopérer, même si des désaccords sur les problèmes à résoudre (Lanzara, 1986), sur les moyens à mettre en œuvre (Béguin, 1994) ou sur les solutions acceptables (Bucciarelli, 1994), sont non seulement probables, mais aussi parfaitement légitimes. Pour influer sur les choix de conception, l'intervention suppose donc une construction sociale, qui "vise à positionner l'ergonome par rapport aux différents acteurs du processus de conception, et à lui permettre de développer avec eux les interactions pertinentes » (Daniellou, 2004).

1. Du type « bon, et bien là le type monte dans la voiture... Et puis dans ce cas-là... » etc. 
Conjointement à cette construction sociale, l'intervention suppose également une compétence technique. Plus l'ergonome veut influer sur l'activité des acteurs de la conception, et plus il doit s'inscrire en amont du projet. En effet, la conception est un processus de détermination progressif d'un objet ou d'une situation de travail et des actions des acteurs de la conception (Terssac, de \& Friedberg, 1996). Mais plus l'intervenant s'inscrit en amont du projet, et plus il lui faut anticiper le fonctionnement et l'activité future : peu ou prou, l'apport de l'ergonome repose donc sur sa capacité à réaliser des anticipations pour orienter la conduite du projet.

Pour réaliser cette anticipation, l'ergonome dispose d'un ensemble de savoirs disciplinaires. Les savoirs généraux sur le fonctionnement de l'homme (vision, auditions, fonctionnement cognitif, etc...) et les savoirs plus spécifiques sur l'adaptation des dispositifs techniques à l'homme, souvent obtenus par des démarches expérimentales (Human Factors ou normalisation) sont de ce type. Ces connaissances sont très importantes car elles permettent de définir des seuils, des conditions limites au-delà desquelles les contraintes seront inacceptables. Néanmoins, elles ne permettent pas de rendre compte de l'activité, c'est-à-dire du caractère intégré et finalisé des conduites humaines dans les situations réelles. Identifier par exemple les caractéristiques de l'audition humaine ne permettra pas de savoir que pour un mécanicien, le « bruit» de la machine est un signal qui lui permettra de garantir la qualité de l'usinage. Ce n'est qu'une fois l'activité anticipée qu'il sera possible, étant donné le fonctionnement humain, de rechercher des solutions adaptées. Pour mettre en œuvre cette démarche dans la conception, Maline (1994) propose d'articuler deux formes d'analyse: les «analyses prospectives » et les « analyses projectives ».

L'analyse prospective vise à analyser des situations existantes dites de référence (la situation à transformer ou d'autres situations) dont les éléments transposables dans le futur doivent être isolés, ce qui demande de définir des unités d'analyse. Les situations d'action caractéristiques (SAC), définies comme « un ensemble de déterminants dont la présence simultanée va conditionner la structuration de l'activité » (Daniellou, 1992), sont une de ces unités. Elles peuvent également être définies comme des unités de tâche transposables aux situations futures, plusieurs SAC articulées entre elles permettant de constituer des scénarios. En outre, l'analyse dans les situations de référence peut aussi chercher à identifier des unités d'action transposables aux situations futures. Un travail récent (Février Quesada, Darses, \& Lewkowicz, 2003) de conception d'un collecticiel permet d'illustrer cette approche. Les auteurs, proposent de recueillir deux classes de données dans des situations de coopération existantes. La première, appelée « tâches collectives » (point sur un état d'avancement, examen d'un sujet particulier, etc) correspond aux situations d'action caractéristiques. La seconde, basée sur l'identification de «fonctions coopératives élémentaires» (poser une question, exprimer un point de vue, ...) identifie des « unités (ou briques) élémentaires de réalisation de toutes les actions coopératives » (Février Quesada, Darses, \& Lewkowicz, ibid.).

L'analyse projective tente de réaliser une projection dans la situation future, en modélisant et en utilisant des techniques de simulation. Idéalement, il s'agit de construire un modèle sur la base des résultats de l'analyse prospective. Une fois la phase de modélisation réalisée, la simulation proprement dite peut être mise en œuvre, afin de répondre à des questions telles que : "si on fait telle chose dans la situation future alors que se passe-t-il ? ». Dans le cadre de cet article, il n'est pas possible d'être exhaustif sur le formalisme des modèles et sur les méthodes de simulation (voir par exemple Amalberti, de Montmollin et Theureau (1991), Sperandio et Wolff (2002) sur les modèles et sur leur formalisme, et Béguin et Weill Fassina (1997) pour une discussion sur les méthodes de simulation). Dans la pratique, les démarches de simulation font néanmoins preuve d'une grande modestie théorique et pratique. Elles peuvent consister par exemple en des «simulations langagières » (à partir d'un modèle de la tâche, par exemple un plan ou une maquette, on demande aux opérateurs de verbaliser leur activité future), ou en démarches d'expérimentation avec prototype (il est demandé alors aux opérateurs de mettre en œuvre le prototype dans une situation construite pour l'occasion, dans laquelle l'intervenant analyse l'activité). 
Soulignons qu'un tel programme, tout au moins sous la forme décrite ici, est très proche dans ses principes du programme de l'ingénierie cognitive tel qu'il a été développé par Woods et ses collaborateurs (Woods, 1991). Il s'en distingue cependant son objet. Comme le souligne Van Daele (1997), l'objectif n'est pas de construire un modèle du fonctionnement du sujet à partir d'un modèle cognitif et de considérations générales issues de la psychologie cognitive, mais de construire un modèle de l'activité dans une situation donnée. Néanmoins, dans les deux cas, la simulation vise à réaliser un pronostic.

\section{3.- Les limites de l'anticipation de l'activité future : une conception continuée dans les situations de travail}

Le principe précédent repose sur des données bien établies, et nous en partageons les grandes lignes : adapter le travail à l'Homme, c'est avant tout postuler qu'une connaissance de son activité constitue une base à partir de laquelle peuvent être prises des décisions durant le projet. Mais, l'anticipation de l'activité future n'est ni complètement possible, ni même toujours souhaitable. C'est pourquoi, il nous semble nécessaire de proposer un second principe : il consiste à compter sur l'activité en situation pour rendre la technique plus performante, tant au plan de l'efficacité productive qu'au plan de la santé des opérateurs.

En effet, la volonté de prédire l'activité se heurte aux limites ontologiques (considérations sur la nature des «choses ») et épistémologiques (considérations sur les modalités d'une connaissance scientifique de ces « choses ») des techniques de simulation. Il existe en effet de fortes limites épistémologiques à la prédiction qui portent, entre autres, sur la clôture du système : quels éléments peuvent raisonnablement être éliminés et / ou lesquels doivent être nécessairement conservés de la situation simulée ? Salembier et Pavard (2004) décrivent les évolutions d'un programme de recherche qui vise explicitement à traiter cette question. À cette difficulté épistémologique, s'ajoute une interrogation ontologique : il s'agit de prédire l'activité d'un sujet susceptible d'inventivité et de créativité. À notre connaissance, cette seconde limite est beaucoup moins discutée dans les travaux sur la simulation, bien que fortement thématisée au sein de la discipline. Déjà Ombredane et Faverge argumentaient en 1955 que «si certains aspects significatifs de la tâche sont prévus, il en est d'autres, en nombre indéfini, qui sont imprévus, et sujets à la découverte du travailleur » (p. 15). Depuis, l'inventivité des sujets en situation n'a cessé d'être soulignée, et Weill-Fassina, Rabardel et Dubois (1993) ont synthétisé cette position, en écrivant que « les actions ne sauraient être réduites à l'effection de réponses à des stimuli reçus, plus ou moins passivement [...] les opérateurs explorent, interprètent, utilisent, transforment leur environnement technique, social, et culturel » (p. 21). Position qui recouvre en grande partie le débat, classique en ergonomie et en psychologie du travail, sur l'écart entre travail prescrit et travail réel. La prescription est une anticipation de nature cognitive et sociale (plus ou moins précise) réalisée par les acteurs de la conception, une pré-écriture un « déjà décidé » qui n'est jamais complètement mis en œuvre. Les éléments qui influencent l'activité de l'opérateur en situation, et que celui-ci retient comme étant significatifs, peuvent varier considérablement d'une situation à une autre ou d'un moment à un autre. L'opérateur rencontre, durant l'exercice de son travail une variabilité souvent « irréductible » (Garrigou, Thibault, Jackson, \& Mascia, 2001), mais aussi des inattendus et des résistances, liés aux contingences de la situation et aux fluctuations de son propre état, lesquels permettent d'argumenter que l'activité est «situé » (Wisner, 1995).

Ces considérations sur les limites de la prédiction signifient d'une part que l'anticipation est forcément limitée et, d'autre part, qu'une anticipation trop forte peut être problématique : des techniques flexibles, qui laissent des marges de décisions, d'autonomie, de responsabilité aux opérateurs seront préférables, dans la mesure où elles rendront les systèmes de travail plus efficients, tant du point de 
vue de la production que des conditions de travail. Nous distinguerons deux types de travaux dont l'enjeu est de rendre opérationnelle, dans la conception, cette flexibilité des techniques : ceux orientés vers la recherche d'une flexibilité diachronique (le système évolue dans le temps après le processus de conception), ceux qui ont pour visée une flexibilité synchronique (concevoir des systèmes de travail qui laissent des « marges de manœuvre » aux opérateurs à un moment donné).

La recherche d'une plasticité diachronique est plutôt mise en avant dans les travaux qui font référence aux courants théoriques de la cognition et de l'action située. Il faut donc « concevoir pour un usage indéterminé » (Robinson, 1993). Gerson et Star (1986) ont jeté les bases d'un tel programme, en argumentant que le problème est de concevoir des systèmes socio-techniques qui réévaluent continuellement leur propre fonctionnement et qui se restructurent eux-mêmes au fur à mesure des circonstances. Dans des situations où il est possible de construire collectivement le contexte de l'action par le langage, Robinson (Op. cit.) identifie un ensemble de spécifications techniques que doivent présenter ce qu'il appelle des « common artefacts ». Ceux-ci doivent faciliter une vigilance périphérique («peripheral awareness »), constituer le cadre informationnel de la communication, permettre, lorsque c'est nécessaire, une double communication (« indexicale » et « langagière »), etc. Dans des contextes d'usage plus individuel, l'idée a été développée de concevoir des artefacts modifiables par l'utilisateur. Mackay (1988) par exemple, s'intéresse à un logiciel nommé " information lens », conçu comme un agent intelligent qui filtre les messages électroniques (Malone et al., 1986) mais qui offre aussi la possibilité à chaque opérateur de constituer son propre filtre compte tenu de ses propres besoins en informations. Les utilisateurs développent donc des fonctionnalités nouvelles qui permettent d'archiver les messages dans une zone appropriée plutôt que comme un filtre. De manière plus générique, Henderson et Kying (1991) ont proposé de définir des niveaux de transformation des systèmes (non modifiables, modifiables et adaptables dans des limites et perspectives envisagées par le concepteur, transformables dans des perspectives nouvelles du point de vue des fonctions) et les pratiques des utilisateurs y correspondent (le choix entre des options préalablement déterminées au cours de la conception initiale, la construction de nouveaux comportements de l'artefact à partir d'éléments existants, etc).

Les travaux orientés par la recherche d'une plasticité synchronique sont bien décrits par Daniellou (2004) : «L'enjeu de l'approche de l'activité future n'est pas de prévoir en détail l'activité qui se déroulera dans l'avenir, mais de prévoir "l'espace des formes possibles d'activité future ", c'est-àdire d'évaluer dans quelle mesure les choix de conception permettront la mise en place de modes opératoires compatibles avec les critères retenus, en terme de santé, d'efficacité productive, de développement personnel. Cette flexibilité permettra d'une part de mieux prendre en compte la diversité et la variabilité des situations et des opérateurs. Elle permettra d'autre part aux opérateurs concernés de pouvoir alterner les modes opératoires $»$. Dans cette approche, un des enjeux de l'analyse prospective est d'identifier les sources de variabilité, afin de vérifier, durant la phase projective, si, étant donné les propositions des concepteurs, les opérateurs pourront atteindre les objectifs de production sans mettre en jeu leur santé. Mac Lean et McKerlie (1995) développent une perspective proche, basée cette fois sur la psychologie cognitive. Ils proposent de mener une " analyse de l'espace de conception » (design space analysis) tout au long du processus de conception. L'objectif de cette analyse n'est pas de contribuer à la définition ou aux spécifications d'un artefact, mais de vérifier que l'« espace de conception » offre suffisamment de flexibilité pour que l'opérateur dispose des marges de manœuvre nécessaires à la réalisation de l'action.

Plus récemment, Vicente (1999) a fait un ensemble de propositions, qui peuvent être interprétées comme la recherche simultanée d'une flexibilité diachronique et synchronique. Vicente souligne tout d'abord qu'il faut « laisser aux travailleurs la possibilité de finir la conception » et que l'artefact doit permettre une adaptation continue de la structure et de l'état du système socio-technique pour faire face aux contingences locales. Mais simultanément, il considère que l'anticipation et la façon dont elle va s'inscrire dans l'artefact, devient une ressource pour l'opérateur, qui l'aidera à orienter 
son action. Ainsi, l'artefact doit présenter des «limites» de fonctionnement, au double sens du terme. Le fonctionnement doit d'une part être clairement identifiable par l'usager, et le guider. En particulier il doit fournir à l'opérateur un «modèle conceptuel» du domaine professionnel ce qui demande une solide analyse de la tâche et des conceptualisations du domaine. D'autre part, le système doit spécifier des frontières à l'action (specifying boundaries on action). Vicente, qui est un spécialiste des systèmes à risques, considère que la spécification de ces frontières consiste à isoler les systèmes de travail des contingences de l'environnement. Mais ceci est plus ou moins possible : par exemple, l'activité d'un conducteur de métro est beaucoup plus isolée des contingences de l'environnement que celle d'un chauffeur de bus.

\section{4.- Un développement conjoint des situations et des personnes}

De notre point de vue, l'approche précédente représente une avancée considérable dans la mesure où elle assume que l'efficacité des dispositifs ne repose pas uniquement sur les artefacts ou les organisations issues des bureaux d'études ou des méthodes, mais également sur l'activité en situation. En outre, elle en tire des conséquences opératoires pour la conception. Mais, paradoxalement, il semble pertinent de se demander si elle prend suffisamment en compte l'activité des opérateurs.

Première remarque : l'hypothèse de plasticité des systèmes techniques peut conduire à sous-estimer le rôle de la technique dans la détermination de l'action. En effet, même si, dès les années 70 , on a montré que des techniques similaires sont employées dans différentes organisations du travail, il n'en reste pas moins que tous les choix organisationnels théoriques ne sont pas possibles dans la mesure où certains de ces choix sont déjà inscrits dans la technique.

Seconde remarque : pour certaines théories de l'action et de la cognition situées, le fait que les opérateurs fassent preuve d'inventivité en situation, qu'ils n'utilisent pas les dispositifs techniques comme leurs concepteurs s'y attendaient, les modifient momentanément ou durablement, est imputé aux caractéristiques des situations. Ainsi, Suchman (1987, p. 50) écrit : «J'introduis l'expression "action située" pour souligner que tout cours d'action dépend de façon essentielle de ses circonstances matérielles et sociales ». De la même manière, Vicente (op. cit) souligne que « finir la conception est une expression qui réfère à l'adaptation continuelle des structures et des états du système socio-technique aux contingences locales particulières ». Mais cette analyse semble trop restrictive car elle réfère trop fortement l'activité à des variables extérieures à l'opérateur (diversité des situations, contingences locales, circonstances matérielles ou sociales, ...). Comme l'a récemment rappelé Leplat (2000) l'activité est certes fonction de la tâche, mais également du sujet. Le cadre des activités avec instruments (Rabardel, 1995 ; Béguin et Rabardel, 2000) rend bien compte de cette double détermination de l'activité, par l'artefact et par les ressources propres de l'opérateur. Dans cette approche, l'instrument est compris comme une entité composite, formé d'une part de l'artefact, dans ses aspects de structure et de forme, et, d'autre part, d'une composante liée au sujet que Rabardel (ibid.) propose de définir comme un schème. Par exemple une clef anglaise ne forme pas un instrument en elle-même. Du fait de ses propriétés (de dureté et de masse,...), cette clé peut être utilisée comme un marteau. Dans cet exemple simple, l'activité dont témoigne l'opérateur n'est pas seulement liée à la variabilité de la situation ou à la fluctuation des circonstances. Elle témoigne d'une dimension ontologique de l'activité que Samurçay et Rabardel (1995) ont proposé d'appeler activité constructive : la constitution, par l'opérateur, des ressources de son action et de son développement. L'activité constructive peut concerner les genèses instrumentales (soit par l'attribution d'une fonction nouvelle à l'artefact, comme dans l'exemple ci-dessus, soit par la modification des ressources opératoires des sujets -les schèmes-). Mais elle peut également concerner les compétences et particulièrement les « concepts pragmatiques » (Samurçay \& Pastré 1995), ainsi que les formes subjectivement organisées de l'action au sein des collectifs, telles que les genres (et leurs recréations individuelles : les styles) (Clot, 1999). 
Bref, toute technologie étant in fine mise en œuvre par les travailleurs, ces derniers doivent développer, dans l'activité constructive, les ressources de leur propre action. En ce sens, les opérateurs contribuent à la conception sur la base de leur singularité et de leur spécificité. C'est pourquoi, nous pensons que l'activité constructive des opérateurs doit faire partie intégrante des processus de conception, et même, que l'inventivité dont témoigne l'activité doit être mise en résonance avec les développements des concepteurs. Nous proposons donc un troisième principe. Celui-ci consiste à chercher à articuler dans un même mouvement, le développement des situations (artefact et/ou organisation) par les concepteurs et le développement des ressources de leur action par les opérateurs. Ce principe n'est pas en rupture avec le précédent : l'efficacité des systèmes de travail ne repose pas uniquement sur les artefacts ou les organisations, mais également sur l'activité des opérateurs. Mais il ajoute une dimension à notre sens essentielle : développement des situations et développement des activités doivent être envisagées conjointement durant la conduite du projet.

Sur quelles bases une telle approche peut-elle être mise en œuvre dans la conception ? Ce principe conduit à une conception développementale, et même dialogique (ce qui ne veut pas dire langagière), où le résultat de l'activité de l'un constitue une hypothèse, qui génère dans l'activité d'autrui un apprentissage, susceptible de valider, d'informer ou de remettre en mouvement la production initiale. Tout un ensemble de travaux d'analyse de l'activité des concepteurs, en particulier ceux qui s'intéressent aux aspects collectifs de la conception, (Hatchuel, 1994 et 1996 ; Bødker \& Grønboek., 1996; Béguin, 1994 et 2003) ont montré l'importance de ces processus dialogiques d'« apprentissages croisés ». Ces processus sont à la fois liés au «dialogue avec la situation» (Schön, 1987) et aux dialogues qui s'instaurent entre les acteurs du processus, qui l'un comme les autres « répondent », « surprennent », et présentent des résistances inattendues. Dans ce contexte, le résultat du travail du concepteur est au mieux une hypothèse, qui sera validée, réfutée, ou plus souvent remise en mouvement à partir des apprentissages des autres acteurs du processus. D'où l'idée de favoriser, durant la conception, des apprentissages mutuels entre concepteurs et opérateurs (Kyng, 1995 ; Bødker et al., 1996 ; Bratteteig, 1997 ; Béguin, 2003), où l'opérateur est susceptible d'apprendre à partir du résultat temporaire du travail du concepteur. Mais où, symétriquement, le concepteur peut être conduit à réaliser de nouveaux apprentissages, voire même à réorienter la conduite du projet, à partir de l'activité constructive des opérateurs.

La mise en place de tels apprentissages n'est pas une tâche facile (Bratteteig, Op. cit.). Mais nous pensons que l'ergonome peut y contribuer. C'est cette contribution que nous allons maintenant illustrer. Elle requiert des développements méthodologiques et conceptuels, mais aussi une sérieuse construction sociale.

\section{1.- Simulation et développement}

Signalons d'abord qu'une approche développementale ne remet pas radicalement en cause l'intérêt des techniques de simulation. En revanche, elle en redessine les enjeux. En effet, appréhender la conception comme un processus dialogique, d'apprentissages croisés, conduit à favoriser la mise en place de systèmes apprenants. Or, les démarches de simulation nous semblent pouvoir contribuer à cet objectif, sous réserve d'être spécifiquement développées dans cette perspective. On se contentera de souligner trois points.

Tout d'abord, il s'agit de faire subir au résultat du travail des concepteurs une épreuve de validité dans l'activité des opérateurs. Dans cette perspective, les démarches de simulation doivent permettre une «mise en situation», du résultat du travail du concepteur et de l'activité de l'opérateur. Des démarches utilisant des environnements virtuels, des «tests » sur maquette (« mock-up », version beta), des méthodes de prototypage, des situations d'expérimentation de prototypes sur des sites pilotes, permettent de telles mises en situation. On ne peut pas entreprendre un inventaire de ces techniques dans le cadre de cet article. Celui-ci serait néanmoins particulièrement utile pour deux 
raisons. D'une part, les techniques qui rendent possibles de telles mises en situation sont extrêmement nombreuses. Or, les supports utilisés sont susceptibles d'engendrer des contraintes pragmatiques (Pavard, 1985). Une maquette par exemple, facilite les échanges entre acteurs, mais ne permet pas d'envisager facilement des options alternatives, du fait des contraintes liées à sa fabrication (Maline, 1994). D'autre part, les différentes techniques ne présentent pas la même valeur selon l'état d'avancement du processus de conception. Ce dernier est avant tout un processus de réduction d'incertitude ponctué d'irréversibilités. Du coup, les différentes techniques ne présentent pas les mêmes avantages et inconvénients, dans la mesure où certaines demandent plus de certitude que d'autres. Des méthodes d'expérimentation avec prototype sur site pilote sont par exemple très intéressantes parce qu'elles réalisent au mieux la mise en situation. Mais elles sont souvent trop tardives : lorsqu'on dispose d'un prototype, de nombreux choix ont déjà été réalisés qui seront difficilement remis en cause compte tenu des irréversibilités et du coût qu'entraîne, par propagation, toute modification.

Ensuite, de telles simulations doivent permettre d'anticiper suffisamment les caractéristiques de la situation future pour que l'opérateur puisse réaliser des apprentissages significatifs. C'est en effet sur la base de ces apprentissages que le résultat du travail du concepteur sera remis en mouvement. En ce sens, les techniques de simulation pour la conception gagneraient à être rapprochées des techniques de simulation pour la formation (Béguin et Pastrée, 2002). Une recherche menée sur l'apprentissage de la conduite de centrale nucléaire sur simulateur pleine échelle (Pastré, 1999) met en avant l'intérêt de ces méthodes pour procéder à un retour réflexif durant la phase de « debriefing» qui suit l'action sur le simulateur. Dans le feu de l'action, les opérateurs sont souvent le jouet des événements. Ce n'est qu'après coup, qu'ils peuvent découvrir la logique qui explique l'enchaînement des faits. La phase de debriefing en favorisant, chez l'opérateur, un retour réflexif sur sa propre action à partir de l'enregistrement de traces observables de son comportement (enregistrement vidéo) et de la situation (enregistrement des paramètres) lui permet de produire les conceptualisations nécessaires pour développer sa propre activité et parler sur son activité. Lors de la conception participative d'un élément du poste de commande et de navigation ${ }^{1} \mathrm{~d}^{\prime}$ un avion de combat de la marine nationale, Amalberti et al. (1999) montrent l'effet spectaculaire de la mise en situation suivie de debriefing. En effet, cette démarche, appliquée alors qu'aucune spécification technique n'était disponible, majorait de $42 \%$ la totalité des remarques, formulées par les pilotes expérimentés, jugées pertinentes par les concepteurs. Les dynamiques à l'oeuvre, et qui permettraient d'expliquer de tels résultats sont loin d'être comprises. Mais elles mériteraient d'être mieux appréhendées, y compris pour la conception, puisqu'elles présentent deux avantages : elles aident les opérateurs à développer les ressources de leur action, et leur permet de développer les conceptualisations dont ils besoin pour en parler avec les concepteurs.

Enfin, les démarches de simulation doivent être appréhendées et construites comme des dispositifs d'échange entre acteurs. Ainsi, dans le cadre d'une recherche dans le secteur de la chimie fine (Béguin, 1998), l'expérimentation d'un prototype d'alarme sur site pilote, en vue d'évaluer les conséquences de son introduction, a donné lieu à une simulation pour mettre en relation d'une part les conditions de destruction du produit par les opérateurs et, d'autre part, le comportement du produit dans des conditions qui entraînent une évolution spontanée du processus vers l'explosion. Dans la mesure où les opérateurs portaient des masques à gaz (normes de sécurité dans de telles conditions), leur activité dans la situation de simulation, plutôt assimilable à du théâtre, voire du mime, a permis cependant de montrer aux membres de la direction présents sur le site (conformément aux procédures imposées par les normes SEVESO dans le cas d'un événement critique que nous simulions) ce qui leur poserait problème en cas de destruction, et ont argumenté, par l'action, les difficultés à y

1. Le pilote saisit sur ce dispositif, et avant chaque mission, les coordonnées en latitude, longitude et altitude des différentes étapes du plan de vol. 
faire face! Dans cet exemple, la simulation apparaît comme une démarche qui vise à mettre à jour des réalités du travail (actuel ou futur), mais en amenant autrui à penser, à sentir et à agir selon la perspective de celui qui simule. Un tel résultat est à rapprocher des travaux actuels menés sur l'autoconfrontation croisée (Clot, 2000). Un des points central de cette méthode vise justement à exploiter, durant l'auto-confrontation, les discordances produites par la présence d'un autre, dont la compréhension est différente. Dans la simulation, comme dans l'auto-confrontation croisée, la présence de ces dissonances est une ressource, qu'il faut exploiter pleinement.

\section{2.- Construire un « monde commun»}

Dans ce qui précède, les démarches de simulation reposent sur les développements des concepteurs, lesquels orientent l'activité constructive des opérateurs (même si les premiers sont susceptibles d'apprendre des seconds). Néanmoins, une perspective dialogique inscrit dans une même logique l'inventivité des concepteurs et celle des opérateurs, en considérant que la nouveauté résulte de l'inscription du résultat du travail de l'un dans l'activité de l'autre. Ceci est possible car il n'existe pas de vide technique : l'arrivée d'un nouvel artefact rencontre toujours des constructions qui lui pré-existent, susceptibles alors d'orienter les apprentissages des concepteurs. Dès lors, le processus dialogique renvoie à l'idée de confrontation entre des connaissances différentes, construites à partir de points de vue différents. Nous avons proposé de rendre compte de cette diversité, et de conceptualiser les " points de vue » associés aux connaissances en utilisant le concept de monde tel qu'il a été développé dans l'oeuvre de Cassirer (1929/1972) et de Goodman (1992) (Béguin, 2004).

La notion de monde est tout d'abord une conceptualisation de la notion de «point de vue » au sens de Prieto (1975). Ce dernier argumente qu'un concept qui consisterait à décrire l'objet dans tous ses aspects serait aussi inutilisable qu'un plan qui serait une copie de la géographie, conservant les propriétés de relief, les distances et la topographie. Ainsi dit Prieto, c'est «à son adéquation, non pas à l'objet, mais au point de vue dont dépend sa pertinence que se mesure la vérité d'un concep » (p. 29). Ensuite, un monde est construit et orienté : il ne retient qu'une partie de la réalité et c'est même une condition de son efficacité. De plus, cette réalité réfère moins à sa substance, qu'à sa signification pour le sujet engagé dans le cadre d'une activité finalisée. Dès lors, l'image est laconique, orientée par des buts, mais opérative (Ochanine, 1978). Ainsi, face à un même objet, co-existent différents systèmes de référence, différents mondes. Comme l'a montré Bucciarelli (1994) dans une analyse ethnologique du travail de concepteurs, ce qu'un dessinateur appelle un « bouton d'arrêt d'urgence », sera appelé une «boîte de jonction » par un ingénieur. Les différents acteurs ont, face à un même objet, des conduites spécifiques, un jargon différencié, mais aussi des concepts, des critères de réussite, et même différents systèmes de valeurs. On serait donc tenté de définir un monde comme un ensemble d'implicites conceptuels, axiologiques et praxiques, qui forment système avec les objets de l'action. Enfin, le concept de monde renvoie à celui de stratégie. Goodman (1992) a une phrase qu'on pourrait reprendre ici : «le sujet perçoit autant qu'il dessine ». Le concept de monde postule une créativité située, qui vise à rendre compte de la production de son milieu par le sujet. Des professionnels ne prélèvent par exemple que très peu d'informations sur la situation; mais elle est pertinente, parce que la situation sera mise en adéquation aux critères de l'action.

Ainsi, lors de la conception d'une alarme destinée à des PME/PMI de la chimie fine classées SEVESO, situation évoquée précédemment, nous avons montré que les concepteurs et les opérateurs mobilisaient deux mondes bien distincts. Les opérateurs conduisent au froid, comme ils le disent eux-mêmes. Ils s'éloignent le plus possible des températures hautes du produit. En effet, ce dernier est exothermique : un réchauffement produit un « emballement de réaction » capable de faire exploser l'installation ${ }^{1}$. La conduite au froid n'est cependant pas sans risque : un produit trop froid

1. L'emballement de réaction constitue le principal facteur de mort d'homme dans la chimie fine. 
s'épaissit, pourrait casser certains matériels en verre, le réchauffement du produit est alors nécessaire ce qui est dangereux. Pour gérer ce risque, et conduire l'installation, les opérateurs prélèvent tout un ensemble d'indices sur le produit, des « zébrures », des « marbrures », etc. qui traduisent la présence de représentations opératives, auxquelles correspondent des concepts : les opérateurs parlent de « cristallisation », et « d'amorce » de cristallisation. D'autre part, ils se sont dotés d'instruments spécifiques comme une « feuille de conduite » pour disposer d'une mémoire de l'évolution de la température du processus. En effet, les conséquences diffèrent selon la rapidité avec laquelle on «s'approche $d u$ froid», (plus la diminution de température est rapide, plus la «cristallisation» apparaît à une température élevée). Bref, les opérateurs travaillent dans un « monde du froid», avec ses instruments, ses concepts et ses représentations opératives, organisés en système. Un monde pour l'action, qui donne sa cohérence à la succession des opérations élémentaires qui composent la conduite au froid. Mais un monde produit : la conduite au froid est une stratégie. Un monde du froid qui est bien distinct du «monde du chaud» des ingénieurs, spécialistes des théories de l'emballement et des explosions. Un monde gazeux et explosif, avec ses théories que l'alarme intègre (elle repose sur un modèle stochastique de l'emballement de réaction, issu de la théorie des catastrophes). Un monde lui aussi produit : les ingénieurs passent le plus clair de leur temps dans un bunker, procédant à des explosions (des « essais en grand ») et réalisant sur cette base des observations minutieuses à partir d'instruments spécifiques. Un monde lui aussi construit par et pour l'action, mais où la référence n'est plus la « cristallisation », mais l'emballement de réaction.

Or, entre ces deux catégories d'experts, de la cristallisation et de l'emballement de réaction, les références partagées ne sont pas constituées $a$ priori. Il était pourtant impossible de rester sur un face à face : les opérateurs sont peu outillés pour faire face au monde du chaud des ingénieurs alors qu'un événement non maîtrisable, casse ou panne, est toujours possible. Symétriquement, les ingénieurs sont mal préparés pour concevoir des aides bien adaptées au monde du froid des opérateurs alors même que les incidents (ou " presque accidents ») éprouvés sur le site ont tous été consécutifs à un refroidissement trop important. D'où l'idée de réaliser deux simulations bien distinctes :

- Une première, centrée sur le monde du froid des opérateurs, durant laquelle les concepteurs apprennent des opérateurs car ces derniers ont utilisé l'alarme comme un thermomètre. Étant donné les coûts de développement et les coûts associés à l'étude, il s'agissait d'un thermomètre fort coûteux mais néanmoins utile car les opérateurs conduisaient au $1 / 10^{\text {éme }}$ de degré avec des thermomètres gradués au $1 / 2$ degré ! Les concepteurs tiendront compte de cette réalité : une nouvelle version de l'alarme intégrera un affichage analogique, couplé à un afficheur digital avec mémoire.

- Une seconde, centrée sur le monde du chaud des concepteurs, durant laquelle les opérateurs apprennent des concepteurs. Des situations ont donc été construites, durant lesquelles les opérateurs conduisaient le process avec un liquide inerte (de l'eau), alors que la cinétique thermique du produit était simulée à partir de données issues d'une explosion expérimentale. Ce processus est édifiant pour les personnels du site : il montre qu'étant donné les conditions architecturales et organisationnelles, il est impossible d'éviter un emballement de réaction.

On pourrait finalement résumer notre position en disant qu'il s'agit de construire un monde commun, c'est-à-dire un système de positions différenciées, une cartographie à plusieurs entrées à partir de laquelle l'activité des uns est mise à l'épreuve dans le monde des autres, dans des situations construites pour l'occasion.

\section{3.- Le développement du système d'activité}

La simulation et la notion de monde fournissent des ressources à l'intervenant : elle l'aide à orienter, à outiller et à guider les apprentissages croisés. La conduite de ces apprentissages est néanmoins sous-tendue par une construction sociale. Daniellou (2004) souligne qu'elle vise à définir la place de 
l'ergonome. Dans une approche développementale, elle concerne l'ensemble des acteurs de la conception. Tout d'abord, parce que le développement conjoint des situations et des personnes implique souvent de faire reconnaître les opérateurs comme des acteurs légitimes de la conception, ce qui, le plus souvent, n'est pas le cas. Ensuite, parce que dans le processus de conception, certains acteurs ont la légitimité, attribuée par l'entreprise ou par un processus de représentation, de se substituer aux opérateurs, que ce soit pour débattre des conditions de travail ou pour faire connaître les problèmes qu'ils rencontrent dans leur activité. L'instauration d'un processus dialogique conduit, de fait, à reconfigurer l'espace social de l'ensemble des acteurs participant au processus de conception et nécessite de l'ergonome qu'il s'intéresse aussi à la dynamique évolutive de l'organisation du processus de conception, et aux apprentissages que les acteurs réalisent dans ce cadre. Les concepts de système d'activité et «d'expansive learning » développés par Engeström $(1987,1999 \mathrm{a}$ et b) dans la lignée des travaux de l'école russe sur l'activité, permettent d'envisager cette reconfiguration et les apprentissages qu'elle nécessite dans une perspective essentiellement fonctionnelle (orientée par l'action).

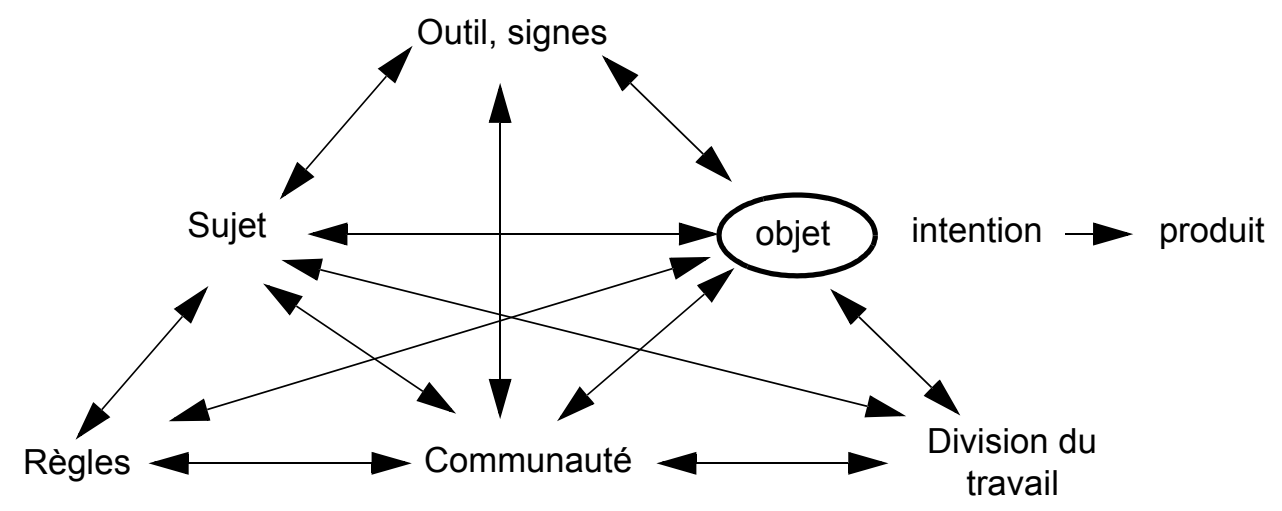

Figure 1.- La structure d'un système d'activité humain (d'après Engeström, 87)

Cette approche historique et culturelle de l'activité met en relation, à travers deux «processus » (Figure 1), un sujet, qui peut-être un collectif, et un objet. Comme le dit Engeström (2001) l'objet n'est pas seulement un matériel brut se prêtant à des opérations logiques faites par le sujet, mais une entité culturelle, une entité interprétée aussi à travers l'action finalisée du sujet. Le premier processus, qui est représenté par le triangle au sommet de la figure, consiste à mobiliser des artefacts, qu'ils soient matériels comme une machine-outil par exemple, ou symboliques comme le langage, des écrans d'ordinateur, pour agir sur l'objet, le transformer, le produire, conformément aux intentions du sujet. Le second, représenté par le triangle orienté vers le bas de la figure, consiste à inscrire l'objet dans une communauté de travail qui participe, avec le sujet, à la transformation ou la production de l'objet. L'ensemble fait système à travers les relations construites d'une part entre cette communauté et le sujet grâce à des règles de travail explicites ou tacites, et, d'autre part, entre cette communauté et l'objet par l'intermédiaire d'une certaine division du travail. Une telle représentation de l'activité permet de saisir simultanément le travail d'un individu ou d'un collectif restreint et son inscription dans une organisation. Il peut aider l'ergonome à comprendre l'espace social du processus de conception ${ }^{1}$ voire éventuellement à aider les acteurs de la conception à en percevoir les limites pour l'instauration d'un processus dialogique.

1. Néanmoins, une telle approche intègre mal les rapports sociaux qui ne sont pas orientés vers la réalisation de l'activité et qui peuvent parfois être prépondérants. 
Ainsi, dans le cas de la conception d'outils d'aide à la lutte contre un pathogène des cultures dans un institut technique agricole, Cerf (2003) met en évidence que le système d'activité qui s'est construit depuis une dizaine d'années autour de cet objet inclut certes les concepteurs de ce type d'artefact, mais mobilise couramment d'autres acteurs. Il s'agit des expérimentateurs de l'institut, des conseillers agricoles qui, en participant au travail d'expérimentation, apprennent l'usage des artefacts, des agriculteurs, chez qui se déroulent parfois les expérimentations, et des ingénieurs régionaux de l'institut qui ont pour mission de faire remonter les besoins des agriculteurs, de participer au développement d'innovations (comme par exemple les outils d'aide à la décision), de faire la promotion de ces innovations auprès des agriculteurs et surtout de leurs conseillers (Chambres d'Agriculture, coopératives agricoles, etc.). Ce «collectif de travail» est régie par une certaine répartition des tâches. Les concepteurs font les modifications sur l'artefact et élaborent les protocoles de test. Les expérimentateurs, qu'ils soient internes à l'institut ou qu'ils travaillent dans des organismes externes, exécutent les protocoles et font remonter les problèmes rencontrés dans la mise en œuvre opératoire ainsi que les données expérimentales. Les ingénieurs régionaux définissent les partenaires qui seront impliqués dans le travail d'expérimentation. Implicitement les règles qui régissent les relations entre le collectif de conception et les autres acteurs de la « collectif», sont que les concepteurs prennent les décisions, mais interagissent avec les autres acteurs pour les prendre.

Ce système d'activité orienté par la conception d'outils d'aide au raisonnement des techniques culturales pré-existe à l'intervention ergonomique. En visant à développer des apprentissages, mais aussi à introduire des utilisateurs comme partie prenante du processus de conception, l'intervention perturbe le système : elle conduit en effet à interroger les règles au sein du collectif, la division du travail, l'usage des essais au champ qui servent jusqu'ici à tester la robustesse et la fiabilité des aides en cours d'élaboration.

L'introduction de nouvelles méthodes d'intégration des mondes des opérateurs (agriculteurs et leurs conseillers agricoles) dans le processus de conception, vient questionner l'expertise des ingénieurs régionaux censés représenter ces opérateurs pour l'institut. Par exemple, en proposant de considérer les conseillers agricoles comme des utilisateurs (et pas uniquement comme des relais d'une innovation) suite à l'analyse de l'activité constructive de ces derniers sur d'autres outils d'aide au raisonnement des traitements fongicides. On questionne également l'expertise des ingénieurs régionaux en structurant la diversité des situations de travail à partir de de la façon dont les opérateurs mobilisent des ressources informationnelles plutôt que sur des critères agronomiques. Cette perturbation s'est concrétisée, dans le cours de l'intervention, par un refus des ingénieurs régionaux de proposer quels pourraient être les agriculteurs et les conseillers qui participeraient à des groupes, permettant un travail conjoint des concepteurs et de ces utilisateurs sur les situations d'usage.

De plus, travaillant sur le développement conjoint des situations et des personnes, l'ergonome vient remettre en cause les protocoles expérimentaux et la place des expérimentateurs : que signifie expérimenter dans cette perspective? Par exemple, comment concevoir les protocoles de telle sorte qu'ils deviennent des supports d'apprentissage, et pas seulement des tests de fiabilité et de robustesse des outils ? Ici, le problème n'a pas réellement émergé car les concepteurs, aidés de l'ergonome, se sont prêtés à cette transformation de leurs actions et à la réalisation d'entretiens avec les utilisateurs. Mais ces entretiens n'ont pas pour autant permis la construction d'une dimension réflexive évoquée plus haut, dans la mesure où les règles entre les acteurs laissaient le pouvoir de décision aux concepteurs qui en retour ont plutôt conduit l'entretien comme un recueil d'informations que comme l'accompagnement d'un travail réflexif.

Comme le suggère Engeström (2001), l'ergonome peut développer avec les acteurs du système d'activité, un processus d'apprentissage qui vise l'expansion du but et de l'objet du système d'activité, contribuant ainsi à une invention collective dont la finalité est de dépasser les dilemmes et les contradictions rencontrées au sein d'un système d'activité ou entre plusieurs systèmes d'activité. 
Selon l'auteur, un tel apprentissage se déroule selon un cycle (Figure 2) et nécessite la mise en évidence de ce qu'il appelle, en réinterprétant les travaux de Vygotski, la zone proximale de développement : c'est-à-dire la distance qui sépare la façon dont le sujet vit son expérience présente et la vision qui en a été construite collectivement (Engeström, 1987). Cette zone n'est pas donnée, elle relève d'un débat au sein d'un ou de plusieurs systèmes d'activité, débat auquel l'intervenant peut contribuer. Concrètement, il s'agit de mettre en évidence les contradictions au sein d'un ou entre systèmes d'activité et de proposer aux acteurs de nouveaux outils conceptuels pour analyser et reconfigurer leur propre pratique (Engeström et al., 1996 ; Seppänen, 2002). On peut se demander si ce mode d'intervention reste dans le champ des compétences de l'ergonome. Cependant les questions qu'il tente de traiter sont à prendre en considération dès lors que l'intervention ergonomique vient modifier l'espace social dans lequel se construit le processus de conception.

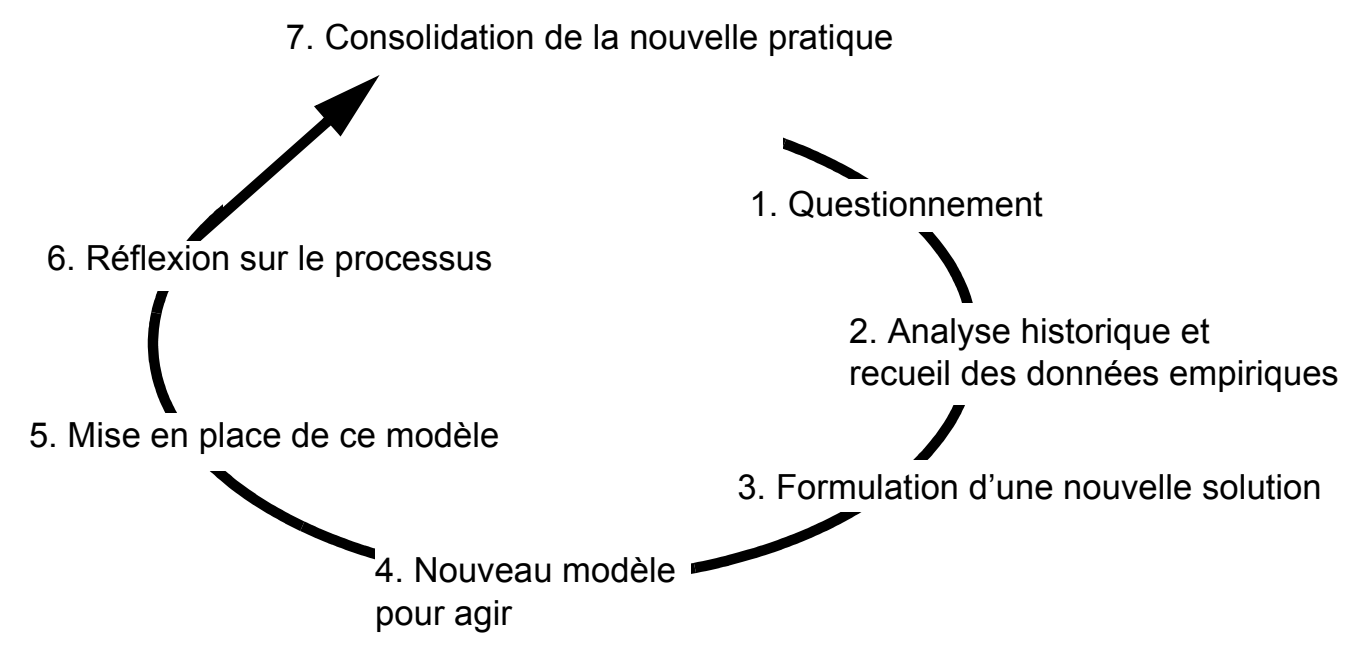

Figure 2.- Le cycle de développement (d'après Engeström, 1999b)

\section{5.- Conclusion}

Nous avons identifié trois postures que l'ergonome peut adopter pour analyser l'activité pour la conception. Il peut chercher à anticiper l'action en situation. Il peut favoriser la conception de dispositifs plastiques, qui orientent (favorisent mais contrôlent) l'action in situ. Il peut encore chercher à saisir et articuler dans un même mouvement le développement des situations de travail et le développement de l'activité des opérateurs.

Ce panorama des formes et des enjeux de la prise en compte de l'activité des opérateurs pour la conception est discutable, probablement incomplet, et donc perfectible. Il ne vise pas à opposer des postures, à stigmatiser certaines ou à en mettre d'autres en valeur. Dans notre esprit, il n'y a d'ailleurs pas de ruptures entre les trois principes que nous distinguons. Il n'y a pas, non plus, de progression d'un principe vers un autre. Distinguer ici, c'est tenter une mise à distance des travaux évoqués, avec le souci de révéler l'existence de positions cohérentes, souvent implicites, mais bien différentes. Un tel exercice montre qu'il existe des paradigmes dans la saisie du travail par l'ergonome (Garrigou, 1995), des traditions différentes dans l'analyse du travail (Clot, 1995), des doctrines distinctes pour la conception des systèmes de travail. Celles-ci engendrent des visions différentes du travail de l'ergonome.

Différences qui portent d'abord sur les connaissances que ce dernier choisit d'introduire dans le processus de conception. Une question essentielle pour lui est de définir la façon dont il construit le rapport du sujet à la tâche : la tâche existe-t-elle indépendamment du sujet et son identification suffit- 
elle à préciser l'activité future ? Faut-il simuler un couplage entre une tâche ainsi comprise et un opérateur modélisé suite à l'analyse de l'activité en situation ? Faut-il créer des situations où l'opérateur peut simuler cette activité future pour aider les concepteurs à comprendre comment faciliter le couplage entre tâche et sujet ? Faut-il aider l'opérateur à construire son environnement de travail à partir des ressources mises à sa disposition? Il reste un travail important pour préciser les limites et intérêts des différentes façons de considérer l'activité future des opérateurs. Il serait en particulier utile de les préciser en relation avec les situations de travail et les processus de conception pour lesquelles elles sont déployées.

En outre, les trois principes évoqués conduisent à des compréhensions différentes de l'acte de conception. La différence la plus importante semble résider dans sa saisie temporelle. Dans la première approche, on anticipe parce qu'on considère qu'à la fin du processus, tout doit être décidé. Dans la seconde on laisse des ouvertures. Adopter une approche misant sur le développement et les apprentissages croisés conduit à appréhender la conception comme un processus cyclique, durant lequel l'apprentissage s'effectue sur des cycles courts (cas envisagés ici), durant lequel conception et développement de l'activité sont appréhendés sur des cycles quasiment synchrones, mais également sur des cycles longs, conception et usage étant alors asynchrones (Henderson, 1991 ; Béguin \& Rabardel, 2000).

Enfin, chacune de ces positions mobilise des acceptions différentes de concepts aussi centraux en ergonomie et en psychologie du travail que «travail prescrit» et «travail réel », "tâche » et " activité ». C'est à notre sens un des intérêts des travaux de recherche menés dans le champ de la conception : ils suscitent un retour sur les concepts les mieux établis de la discipline. La participation concrète des ergonomes dans les processus de conception ne consiste pas simplement à appliquer des principes ou des concepts, ni à s'inscrire dans les démarches de conception. Elle contribue au travail scientifique qui engendre ces concepts, les remet en question, les confronte à la réalité, les soumet aux leçons de l'expérience.

\section{BIBLIOGRAPHIE}

Amalberti, R., Maugey, B., Béguin, P., Reuzeau, F., \& Colas, R. (1999). Enjeux et méthode de la participation des usagers dans la conception des cockpits d'avions civils et militaires : résultats et hypothèses de recherche. Rapport de recherche des projets GIS « Sciences de la cognition » (cognisciences) et « Système de production » (Prosper), MRASH, Lyon.

Amalberti, R., de Montmollin, \& Theureau, J. (Eds.) (1991). Modèles en analyse du travail. Liège: Mardaga.

Béguin, P. (1994). De l'individuel au collectif dans les activités avec instruments. Thèse de doctorat, Paris, Laboratoire d'Ergonomie, CNAM.

Béguin, P. (1998). Simulation et participation. Actes des « Journées de la pratique », Bordeaux, 18, 1920 Mars 1998.

Béguin, P. (2003). Design as a mutual learning process between users and designers. Interacting with Computers. 15/5, 709-730.

Béguin, P. (2004). Monde, Monde commun et version des mondes. À paraître dans le Bulletin de Psychologie.

Béguin, P., \& Weill Fassina, A. (1997). De la simulation des situations de travail à la situation de simulation. In P. Béguin, A. Weill-Fassina. La simulation en Ergonomie : connaître, agir, interagir. Toulouse: Octarès, $5-28$.

Béguin, P, \& Darses F. (1998). Le travail des concepteurs et la conception du travail. Conférence invitée aux deuxièmes journées Recherche et Ergonomie. 9, 10 et 11 Février 1998. Université de Toulouse le Mirail, 23-38. http://www.ergonomie-self.org/Pages/rechergo98

Béguin, P., \& Rabardel, P. (2000). Designing for instrument mediated activity. Scandinavian Journal of Information Systems, Vol.12. 173-190. 
Béguin, P., \& Pastré, P. (2002). Working, learning and designing through simulation. In Bagnara, S., Pozzi, S., Rizzo, A., Wright, P. (eds). Proceedings of the $11^{\text {th }}$ European Conference on Cognitive Ergonomics : cognition, culture and design. 5-13.

Bødker, S., \& Grønboek K. (1996). Users and designers in mutual activity: an analysis of cooperative activities in systems design. In Y. Engeström \& D. Middleton (Eds). Cognition and communication at work. Cambridge: University Press, 87-102.

Bratteteig, T. (1997). Mutual learning. Enabling cooperation on systems design. In Bras K. \& Monteiro E. (eds). Procedings of IRIS 20. University of Oslo, Conf. Proc. N¹, June 97, 1-19.

Bucciarelli, L. L. (1994). Designing Engineers. Cambridge: The MIT Press.

Cassirer, E. (1929/1972). Philosophie des formes symboliques, Paris : Éditions de Minuit.

Cerf, M. (2003). Developing new design methods in a French agricultural R\&D body: learning issues in designer-user network for crop monitoring systems, $19^{\text {th }}$ colloquium of the European Group for Organizational Studies, $3^{\text {th }}-5^{\text {th }}$ July, Copenhagen.

Clot, Y. (1996). Le travail sans l'homme? Pour une psychologie des milieux de travail et de vie. Paris: La découverte (coll. Textes à l'appui).

Clot, Y. (1999). La fonction psychologique du travail. Paris: PUF (coll. Travail Humain).

Clot, Y. (2000). La formation par l'analyse du travail. In B. Maggi (Ed.), Manières de penser, manières d'agir en éducation et en formation, Paris: PUF.

Daniellou, F. (1992). Le statut de la pratique et des connaissances dans l'intervention ergonomique de conception. Thèse d'habilitation à diriger des recherches, Université de Toulouse-Le Mirail, France.

Daniellou, F. (2004). Ergonomie et conduite de projet. In P. Falzon (Coord.), L'ergonomie, Paris: PUF, à paraître.

Engeström, Y. (1987). Learning by expanding : an activity-theoretical approach to developmental research, Helsinki: Orienta-Konsultit.

Engeström, Y., (1999a). Expansive visibilization of work : an activity-theoretical perspective. Computer Supported Cooperative Work, 8, 63-83.

Engeström, Y. (1999b). Introduction, Learning by Expanding, German Edition, 11p.

Engeström, Y. (2001). Expansive Learning at Work : toward an activity theretical reconceptualization. Journal of Education and Work, Vol 14 (1), 133-156.

Engeström, Y., Virkkunen, J., Helle, M., Piihlaja, J., \& Poikela R. (1996). Change Laboratory as a tool for transforming work. Lifelong Learning in Europe, 1(2), 10-17.

Falzon, P. (1995). Les activités de conception : réflexions introductives. Performances Humaines et Techniques, 74, 7-11.

Février Quesada, T., Darses, F., \& Lewkowicz, M. (2003). Une démarche centrée utilisateur pour la conception d'un portail coopératif d'aide à l'innovation. Ingénierie des Systèmes d'Information RSTI série ISI, 8(2), 11-31.

Garrigou, A. (1995). La compréhension de l'activité des concepteurs : un enjeu essentiel pour les ergonomes. Performances Humaines et Techniques, $n^{\circ} 74,2-21$.

Garrigou, A., Thibault, J. F., Jackson, M., \& Mascia, F. (2001). Contributions et démarche de l'ergonomie dans les processus de conception. Pistes, $3 / 2 \mathrm{http} / /$ www.pistes.uqam.ca/v3n2/articles/v3n2a6.htm

Gerson, E., \& Star, S. (1986). Analysing Due process in the work place. ACM Transactions on office information systems, 4/3, 265-287.

Goodman, N. (1992). Manière de faire des mondes. Marseille : J. Chambon.

Hatchuel A, (1994). Apprentissage collectif, et activités de conception. Revue Française de Gestion, Juin, Juillet, Août 94, 109-120.

Hatchuel, A. (1996). Coopération et conception collective. Variété et crises des rapports de prescription In G. de Tersac, E. Friedberg (Eds.). Coopération et conception. Toulouse: Octarès. 
Henderson, A. (1991). A development perspective on interface, design and theory. In J. Carroll (Ed.) Designing interaction : Psychology at the human computer interface. Cambridge: University Press, 254268.

Henderson, A., \& Kyng, M. (1991). There is no place like home. Continuing design in use. In Greenbaum J. et Kyng M. (Eds.), Design at work: cooperative design of computer systems. Hillsdale: Lawrence Erlbaum Associates Publishers.

Kyng, M. (1995). Creatring contexts for design. In Carrol, J. M. (Ed.) Scenario-based design. Envisionning work and technology in system development. New York: John Wiley \& Sons, 85-108.

Lanzara, G. F. (1986). La théorie de la conception entre "problem solving" et "problem setting". In A. Demailly, J. L. Lemoigne (eds.). Sciences de l'intelligence et sciences de l'artificiel. Lyon: P.U.L.

Leplat, J. (2000). L'analyse psychologique du travail en ergonomie. Toulouse: Octarès.

Mac Learn, A., \& McKerlie, D. (1995). Narrowing the specification-implementation gap in scenario based design. In Carrol, J. M. (Ed.) Scenario-based design. Envisionning work and technology in system development. New York : John Wiley \& Sons, 123-145.

Mackay, W. (1988). Mote than just a communication system : diversity in the use of electronic mail. Working paper, Sloan school of management, MIT.

Maline, J. (1994). Simuler le travail, une aide à la conduite de projet. Paris : ANACT.

Malone, T. W., Grant, K.R., \& Turbak, F., (1986). The information LENS: an intelligent system for information sharing and organisations, Proceedings of the CHI 86 Conference on Human Factors in computing systems, Boston.

Nicolas, L. (2000). L'activité de simulation dans l'analyse fonctionnelle : vers des outils anthropocentrés pour la conception de produits automobiles. Thèse de Doctorat d'Ergonomie. Laboratoire d'Ergonomie, CNAM, Paris.

Ochanine, D. (1978). Le rôle des images opératives dans la régulation des activités de travail. Psychologie et Education, Vol. 2, 63-72.

Ombredane, A., \& Faverge, J. M. (1955). L'analyse du travail. Paris: PUF.

Pastré, P. (1999). Comprendre après coup grâce à la simulation. Education Permanente. $n^{\circ} 139,90-115$.

Pavard, B. (1985). Le traitement de texte professionnel : activités cognitives et contraintes pragmatiques. Document du laboratoire d'Ergonomie du CNAM. Paris.

Prieto, L. J. (1975). Pertinence et pratique. Essai de sémiologie. Paris: Editions de Minuit.

Rabardel, P., (1995). Les hommes et les technologies. Approche Cognitive des instruments contemporains. Paris: Armand Colin.

Robinson, M. (1993). Design for unanticipated use. In G de Michelis, C. Simone and K. Schmidt (Eds.). Proceedings of the third European conference on C.S.C.W.-ECSCW'93, Milan. 187-202.

Salembier, P., \& Pavard, B. (2004). Analyse et modélisation des activités coopératives situées. @ctivités, 1 (1), http://www.activites.org/

Samurcay, R., \& Rabardel, P. (1995). Work competencies: some reflections for a constructivist theoretical framework. In proceedings 2nd Work Process Knowledge Meeting: Theoretical approaches of competences at work, Courcelle sur Yvette France, 19-21 octobre 1995.

Samurçay, R., \& Pastré, P. (1995). La conceptualisation des situations de travail, dans la formation des compétences. Education Permanente $n^{\circ} 123,13-31$.

Schön, D. (1987). Educating the reflective practitioner. San Francisco, Jossey Bass.

Seppänen, L. (2002). Creating tools for farmers' learning: an application of developmental work research, Agricultural Systems, vol 73, 1, 129-145.

Sperandio, J. C., \& Wolff, M. (2002). Formalismes de modélisation pour l'analyse du travail et l'ergonomie. Paris: PUF (coll. Le Travail Humain).

Suchman, L. (1987). Plans and situated actions. Cambridge, University Press.

Terssac G. (de), \& Friedberg E. (Eds.). (1996). Coopération et conception. Toulouse: Octarès. 
Thomas, J. \& Kellogg, W. (1989). Minimizing ecological gaps in user interface design, IEEE Software, 78-86.

Van Daele, A. (1997). Contribution de la simulation à l'analyse de l'activité de l'opérateur en sitution dynamique. In P. Béguin \& A. Weill-Fassina. La simulation en Ergonomie : connaître, agir, interagir. Toulouse: Octarès.

Vicente, K. J. (1999). Cognitive work analysis: toward safe productive and healthy computer-based works. Lawrence Erlbaum Associate Publishers.

Weill-Fassina, A., Rabardel, P., \& Dubois, D. (1993). Représentation pour l'action, Toulouse: Octarès.

Wisner, A. (1995). Understanding problem building: Ergonomics Work analysis. Ergonomics, 38, 596-606.

Woods, D. D. (1991). The cognitive systems engeniering. In Helander, M. (Ed.) Handbook of Human Computer Interaction. North Holland: Elsevier Sciences Publisher B.V. 